\title{
Genome-wide identification and transcriptome profiling reveal that E3 ubiquitin ligase genes relevant to ethylene, auxin and abscisic acid are differentially expressed in the fruits of melting flesh and stony hard peach varieties
}

Bin Tan ${ }^{1,2+}$, Xiaodong Lian ${ }^{1,2+}$, Jun Cheng ${ }^{1,2}$, Wenfang Zeng ${ }^{2,3}$, Xianbo Zheng ${ }^{1,2}$, Wei Wang ${ }^{1,2}$, Xia Ye $e^{1,2}$, Jidong Li ${ }^{1,2}$, Zhiqian $\mathrm{Li}^{1,2}$, Langlang Zhang ${ }^{1,2}$ and Jiancan Feng ${ }^{1,2^{*}}$ (i)

\begin{abstract}
Background: Ubiquitin ligases (E3) are the enzymes in the ubiquitin/26S proteasome pathway responsible for targeting proteins to the degradation pathway and play major roles in multiple biological activities. However, the E3 family and their functions are yet to be identified in the fruit of peach.
\end{abstract}

Results: In this study, genome-wide identification, classification and characterization of the E3 ligase genes within the genome of peach (Prunus persica) was carried out. In total, 765 E3 (PpE3) ligase genes were identified in the peach genome. The PpE3 ligase genes were divided into eight subfamilies according to the presence of known functional domains. The RBX subfamily was not detected in peach. The PpE3 ligase genes were not randomly distributed among the 8 chromosomes, with a greater concentration on the longer chromosomes. The primary mode of gene duplication of the PpE3 ligase genes was dispersed gene duplication (DSD). Four subgroups of the BTB subfamily never characterized before were newly identified in peach, namely BTBAND, BTBBL, BTBP and BTBAN. The expression patterns of the identified E3 ligase genes in two peach varieties that display different types of fruit softening (melting flesh, MF, and stony hard, SH) were analyzed at 4 different stages of ripening using Illumina technology. Among the 765 PpE3 ligase genes, 515 (67.3\%) were expressed (FPKM > 1) in the fruit of either MF or SH during fruit ripening. In same-stage comparisons, 231 differentially expressed genes (DEGs) were identified between the two peach cultivars. The number of DEGs in each subfamily varied. Most DEGs were members of the BTB, F-box, U-box and RING subfamilies. PpE3 ligase genes predicted to be involved in ethylene, auxin, or ABA synthesis or signaling and DNA methylation were differentially regulated. Eight PpE3 ligase genes with possible roles in peach flesh texture and fruit ripening were discussed.

(Continued on next page)

\footnotetext{
* Correspondence: jcfeng@henau.edu.cn

${ }^{\dagger}$ Bin Tan and Xiaodong Lian contributed equally to this work.

${ }^{1}$ College of Horticulture, Henan Agricultural University, Zhengzhou 450002,

China

${ }^{2}$ Henan Key Laboratory of Fruit and Cucurbit Biology, Zhengzhou 450002,

China

Full list of author information is available at the end of the article
}

(c) The Author(s). 2019 Open Access This article is distributed under the terms of the Creative Commons Attribution 4.0 International License (http://creativecommons.org/licenses/by/4.0/), which permits unrestricted use, distribution, and reproduction in any medium, provided you give appropriate credit to the original author(s) and the source, provide a link to the Creative Commons license, and indicate if changes were made. The Creative Commons Public Domain Dedication waiver (http://creativecommons.org/publicdomain/zero/1.0/) applies to the data made available in this article, unless otherwise stated. 
(Continued from previous page)

Conclusions: The results of this study provide useful information for further understanding the functional roles of the ubiquitin ligase genes in peach. The findings also provide the first clues that E3 ligase genes may function in the regulation of peach ripening.

Keywords: Ubiquitin protein ligase gene family, The UPS pathway, Peach (Prunus persica), Flesh texture, Fruit ripening

\section{Background}

The ubiquitin/26S proteasome pathway in eukaryotes affects nearly all aspects of growth and development [1] through the regulated degradation of proteins. The ubiquitin/26S proteasome pathway consists of three key enzymes, namely an E1 (the ubiquitin-activating enzyme), an E2 (the ubiquitin-conjugating enzyme) and an E3 (the ubiquitin ligase). An E3 protein is responsible for selecting and then transferring the E2-bound ubiquitin to the target protein. The number of E1s, E2s and E3s varies greatly among various plants. For example, Arabidopsis has two E1s, 47 E2s and more than 1300 potential E3s. Rice has six E1s, 49 E2s and more than 1300 E3s [2]. E3 proteins are the most numerous components within the ubiquitin/26S proteasome pathway, due to their role in interacting with the multitude of targets in the plant.

The E3 superfamily comprises single subunit proteins and cullin-based multi-subunit protein complexes, both of which can mediate ubiquitination [3, 4]. Different E3s recognize specific substrates. The nine E3 subfamilies include the RING, HECT, U-box, F-box, cullin, BTB, DDB, RBX and SKP subfamilies. Members of the RING, HECT and U-box subfamilies can function as a single subunit, while SCF (SKP1, Cullin1 and F-box protein), CUL3-BTB and CUL4-DDB proteins function in multisubunit protein complexes. In these complexes, the Fbox or BTB proteins recognize substrates. Many of the subfamilies of the E3 proteins have been extensively studied, see the reviews [5-7].

The establishment of the plantsUPS (a database of plant proteins related to the ubiquitin proteasome system) enables the exploration and comparative analysis of E3 ligase genes in higher plants (http://bioinformatics. cau.edu.cn). The genome-wide identification and classification of E3 ligase genes is already available in a few plants, including Arabidopsis (Arabidopsis thaliana), rice (Oryza sativa), poplar (Populus trichocarpa), soybean (Glycine max), grape (Vitis vinifera), Medicago truncatula, and maize (Zea mays) [2].

Peach (Prunus persica L.) is a major commercial stone fruit grown worldwide, with its center of origin in China. Fruit texture is an important characteristic of peach. Fruit texture affects not only peach storage and processing, but also consumer preference. Peach is a climacteric fruit that undergoes changes in ethylene production and firmness during ripening. According to the changes in fruit firmness and texture during ripening, peach cultivars are divided into three groups, including melting flesh (MF), non-melting flesh (NMF) and stony hard (SH) [8]. Previous studies have shown that endopolygalacturonase (endo-PG), the enzyme responsible for cleaving the polygalacturonan network (pectins) of the cell walls, is a candidate gene for controlling the MF/ NMF flesh texture [9-11]. SH fruits barely exhibit softening on the tree or after harvest and remain crisp due to a lack of ethylene production [12-14]. PpYUCCA11 (YUCCA Flavin mono-oxygenase gene), a candidate gene for the $\mathrm{SH}$ phenotype in peach, regulates auxin biosynthesis during peach fruit ripening $[12,15]$. In addition to auxin and ethylene, abscisic acid (ABA) also plays critical roles in fruit ripening [16-18]. In peach, ABA appears to regulate ripening by affecting ethylene, cell wall and auxin-related genes, while ethylene acts as feedback regulator of $\mathrm{ABA}$, contributing to rapid fruit softening $[16,19]$.

E3 proteins participate in numerous plant processes, including the light response [20], abiotic stress response $[21,22]$, flower and fruit development $[23,24]$, and hormone perception and signaling $[25,26]$. In this study, the ubiquitin protein ligase genes from the peach genome database (https://www.rosaceae.org/) were identified. The chromosomal locations, gene duplication and phylogenetic analysis of each PpE3 were analyzed. The in situ-translated proteins were analyzed based on signature E3 domains. The transcriptome profiles of the PpE3 ligase genes from both a MF ('Zhongyoutao 13') and a SH ('Zhongyoutao 16') cultivar were examined during fruit ripening. Our aims were to identify what roles specific PpE3 ligases may play during ripening process of peach fruit.

\section{Results \\ Identification and chromosomal distribution of E3 ubiquitin ligase genes in peach}

In peach, 765 PpE3 ligase genes were identified through BLAST analysis using protein sequences from Arabidopsis and grape against the peach genome database (Additional file 1: Table S1). The PpE3 ligase genes account for almost $3.0 \%$ of the predicted proteins in the peach genome. The number of putative E3 ligase genes 
in peach was greater than identified in $V$. vinifera (677) but was significantly less than the numbers in six other species (Table 1).

Normally there are nine subfamilies in the E3 ligase gene family. Only eight E3 subfamilies were identified in the peach genome, namely the BTB, Cullin, DDB, F-box, HECT, RING, SKP and U-box subfamilies. The RBX subfamily was not found in peach. The number of genes in the different subfamilies differed in peach. The largest number of genes, 338, was in the RING subfamily. The second largest was 267 in the F-box family. The RING and F-box subfamilies represented $79 \%$ of the predicted PpE3 ligase genes. The smallest subfamily was the DDB subfamily, with only three genes identified in peach.

For each of the PpE3 ligase genes, the exons, introns, additional domains and the length of each domain were analyzed (Additional file 1: Table S2). Most of PpE3 ligase genes contained introns, with numbers varying from 0 to 30. According to the number of introns, the PpE3 ligase genes were divided into 5 groups. Most of the PpE3 ligase genes, 408, contained between 1 and 5 introns. Of these genes, 191 genes lacked an intron in the second position. Only four PpE3 ligase genes, three in the RING and one in the HECT subfamily, have more than 20 introns. The number of introns in different subfamilies might have some relationship with their different functions.

All 765 of the identified PpE3 ligase genes were mapped onto one of the eight peach chromosomes (Additional file 2: Fig. S1). The largest number of genes (173) was located on chromosome 1, including 16 BTB, four Cullin, 78 F-box, two HECT, 61 RING, one SKP and $11 \mathrm{U}$-box genes. Only 68 genes were found on chromosome 8 (Additional file 1: Table S3). Members of the BTB, U-box, F-box and RING subfamilies could be found on every chromosome, while there was no Cullin gene on chromosomes 2, 6, or 7, no SKP gene on chromosomes 3, 5, or 6 , no HECT gene on chromosomes 2 , 3 , or 5 , and no DDB gene on chromosome 1, 3, 4, 5, or 8. The more abundant E3 ligase gene subfamilies were mainly present on the longer chromosomes (Chr 1 and
Chr 6). This result indicated that the PpE3 ligase genes are not evenly distributed on each chromosome.

\section{Gene duplication pattern analysis}

To explain the expansion and evolution of the E3 ligase gene family in peach, the patterns of gene duplication were analyzed and compared across the peach genome (Table 2). Dispersed gene duplication (DSD, 48\% of 765) was responsible for the largest number of gene duplications among the PpE3 ligase genes, followed by tandem duplication (TD, 20\%) and whole-genome duplication (WGD, 19\%). However, the expansion of the PpE3 subfamilies did not all follow the same patterns. For the BTB subfamily, the largest number of gene pairs was derived from DSD (51\% of 67), followed by WGD (21\%), TD (16\%) and proximal pairs (PD, 4\%). DSD was also the most common duplication mode for the F-box (39\% of 267 ), HECT (100\% of 7), RING (53\% of 338), SKP (44\% of 16), and U-box (59\% of 54) subfamilies. WGD was the second most common mode of duplication for the RING (27\%), SKP (31\%), U-box (30\%) and Cullin (30\%) subfamilies. In the F-box subfamily, TD (37\% of 267) was the second most common mode of gene duplication. No duplicated gene pairs were detected in the DDB subfamily.

The genomic distribution of the different types of gene duplications found in the PpE3 family was dissected (Fig. 1; Additional file 1: Table S4). The DSD, TD, WGD, PD and singleton patterns of each subfamily are shown in Fig. 1 as concentric layer 0 , layer 1 , layer 2, layer 3 and layer 4, respectively. Each syntenic pair is linked by a colored line, with the colors representing the different subfamilies. The DDB subfamily contained only singleton genes. DSD (layer 0) is the most prevalent gene duplication pattern in each subfamily and was found on each peach chromosome. The other patterns of gene duplication, including TD, WGD, PD and unduplicated singletons, in each subfamily occurred randomly among the different chromosomes. These results provide further insights into the expansion of the PpE3 family in peach.

Table 1 Number of E3 ubiquitin ligase genes, by subfamily, in peach and seven other plant species

\begin{tabular}{|c|c|c|c|c|c|c|c|c|c|c|}
\hline & RING & F-box & BTB & U-box & SKP & Cullin & HECT & $\mathrm{DDB}^{\mathrm{a}}$ & $\mathrm{RBX}^{\mathrm{a}}$ & Total \\
\hline Peach & 338 & 267 & 67 & 54 & 16 & 13 & 7 & 3 & $\mathrm{NI}$ & 765 \\
\hline Arabidopsis ${ }^{\mathrm{b}}$ & 465 & 654 & 79 & 62 & 21 & 10 & 7 & 5 & 2 & 1354 \\
\hline Grape $^{b}$ & 330 & 153 & 63 & 56 & 10 & 8 & 9 & $\mathrm{NI}$ & $\mathrm{NI}$ & 677 \\
\hline Rice $^{b}$ & 378 & 702 & 126 & 76 & 27 & 10 & 8 & 3 & 2 & 1387 \\
\hline Soybean ${ }^{b}$ & 725 & 418 & 77 & 120 & 18 & 24 & 19 & 3 & 2 & 1519 \\
\hline Poplar ${ }^{b}$ & 399 & 335 & 81 & 93 & 15 & 13 & 7 & 5 & 3 & 1027 \\
\hline Medicago $^{b}$ & 294 & 539 & 41 & 38 & 36 & 12 & 9 & $\mathrm{NI}$ & $\mathrm{NI}$ & 1011 \\
\hline Maize ${ }^{b}$ & 401 & 325 & 129 & 119 & 31 & 6 & 21 & $\mathrm{NI}$ & $\mathrm{NI}$ & 1100 \\
\hline
\end{tabular}

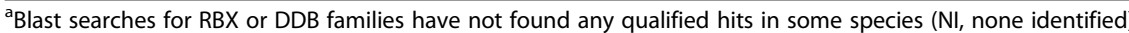

${ }^{\mathrm{b}}$ The data were collected from the plantsUPS database (http://bioinformatics.cau.edu.cn) [2] 
Table 2 The mode of duplication for the gene pairs in each E3 ligase subfamily in peach

\begin{tabular}{lllllll}
\hline Subfamily & \multicolumn{2}{l}{ Modes } & & & & Total \\
\cline { 2 - 5 } & DSD & TD & WGD & PD & Singleton & \\
\hline BTB & 34 & 11 & 14 & 3 & 5 & 67 \\
Cullin & 3 & 3 & 4 & 3 & & 13 \\
DDB & & & & & 3 & 3 \\
F-box & 103 & 98 & 12 & 38 & 16 & 267 \\
HECT & 7 & & & & & 7 \\
RING & 179 & 34 & 92 & 15 & 15 & 338 \\
SKP & 7 & & 5 & 4 & & 16 \\
U-box & 32 & 5 & 16 & 0 & 1 & 54 \\
Total & 365 & 151 & 143 & 63 & 43 & 765 \\
\hline
\end{tabular}

DSD: dispersed gene duplication; PD: proximal duplication; TD: tandem duplication; WGD: whole genome duplication. 'null' represents no finding of that gene duplication pattern within a subfamily

\section{Classification and phylogenetic analysis}

SMART and Pfam databases were used to detect the specific domains, shared domains, and other domains of the predicted PpE3 proteins in the eight subfamilies in peach. All E3 proteins in peach carried their subfamilyspecific domain. In all, 81 other types of domains were identified in five of the E3 subfamilies (BTB, F-box, Ubox, RING and HECT), some of which appeared in more than one subfamily. The classification of the BTB, F-box and U-box genes was based on the subfamily-specific domains plus additional domains.

According to domains present in the BTB proteins, the BTB subfamily could be divided into 14 subgroups (Fig. 2), with 1 to 22 genes in each subgroup (Additional file 1: Table S5). Notably, 21 BTB genes containing the NPH3 domain were detected in BTBN subgroup. Four new subgroups of BTB proteins with different combinations of domains were identified in peach and named BTBAND (BTB-ANK-NPR-DUF), BTBBL (BTB-BACKLRR), BTBP (BTB-Pentapeptide) and BTBAN (BTBANK-NPR). The observation of these new BTB subgroups in peach implies that these genes might play novel functions during the growth and development of peach. The phylogenetic analysis results of the BTB subfamily are shown in Fig. 3. Most subgroups were clustered together, such as the BTBN, BTBM and BTBT subgroups. The results were consistent using both the SMART and Pfam databases.

F-box proteins contain different domains and are classified into differing numbers of subgroups in different species. In peach, the FBX subgroup had 128 members with no other identified domains except the F-box motif. The other nine subgroups were named according to a previous study [27]. The numbers of genes in the different subgroups were quite different, with 64 in FBA, 19 in FBD, 2 in FBDL, 14 in FBL, 12 in FBK, 6 in FBP, 2 in
FBW, 8 in FBDUF and 12 in FBO (Additional file 2: Fig. S2; Additional file 1: Table S5). Most members of some subgroups, such as FBX, FBA, FBP, FBK, FBL, and FBD, clustered together. Members of the FBO subgroup were scattered about the tree, possibly due to the non-uniform domains in the FBO subgroup (Additional file 2: Fig. S3; Additional file 1: Table S5).

The U-box proteins of peach were also divided into seven subgroups, according to the identity of the additional domain (Additional file 2: Figure S4; Additional file 1: Table S5). The U-box subgroup has 15 members that carry only the U-box domain without any other specific domains. Twenty-five U-box proteins contained the Ubox domain and the ARMADILLO (ARM) domain, which was present in 1 to 6 repeats. Eight PpE3 proteins contained Pkinase domain. The TPR (TetratricoPeptide Repeat), UFD2 (Ubiquitin Fusion Degradation 2), and KAP (Kinesin-associated protein) subgroups each contained one member, and the WD40 subgroup had three members. In the phylogenetic tree of the U-box proteins, members of the ARM, U-box, Pkinase and WD40 subgroups clustered together (Additional file 2: Figure S5; Additional file 1: Table S5). The phylogenetic analysis of the eight subgroups partially supports our classification based on SMART and Pfam domains analyses.

There were 352 RING domains found in the 338 predicted proteins identified in the peach genome (Additional file 2: Figure S6; Additional file 1: Table S5). According to the spacing between the amino acids that bind the metal ligands or substitutions at one or more of the metal ligand positions, the RING subfamily was classified into six subgroups, including the RING-C2 (18), RING-G (3), RING-HC (198), RING-H2 (109), RING-S/ $\mathrm{T}$ (4) and RING-v (20) subgroups. In the phylogenetic tree, members of most subgroups clustered together, with the exception of the proteins Prupe.1G303200 and Prupe.5G119800, two members of the RING-S/T subgroup that clustered with the RING-HC subgroup (Additional file 2: Fig. S7; Additional file 1: Table S5). This may be because these two genes evolved from the RING-HC subgroup.

The C-terminal end of the HECT proteins from peach contained an approximately 350-amino acid HECT domain (Additional file 1: Table S5). Proteins in the HECT subfamily also contained other domains, such as UBA (Ub-associated) and UIM (Ub-interacting), both of which are potentially important for ubiquitin ligase function. Based on the presence of additional protein motifs as predicted by the SMART and Pfam database, the HECT subfamily could be divided into three subgroups: (i) Subgroup I, only containing the HECT domain (4); (ii) Subgroup II, containing UBA, UIM and DUF domains (2); and (iii) Subgroup III, containing a UBQ (Ubiquitin homologues) domain (1) (Additional file 2: 


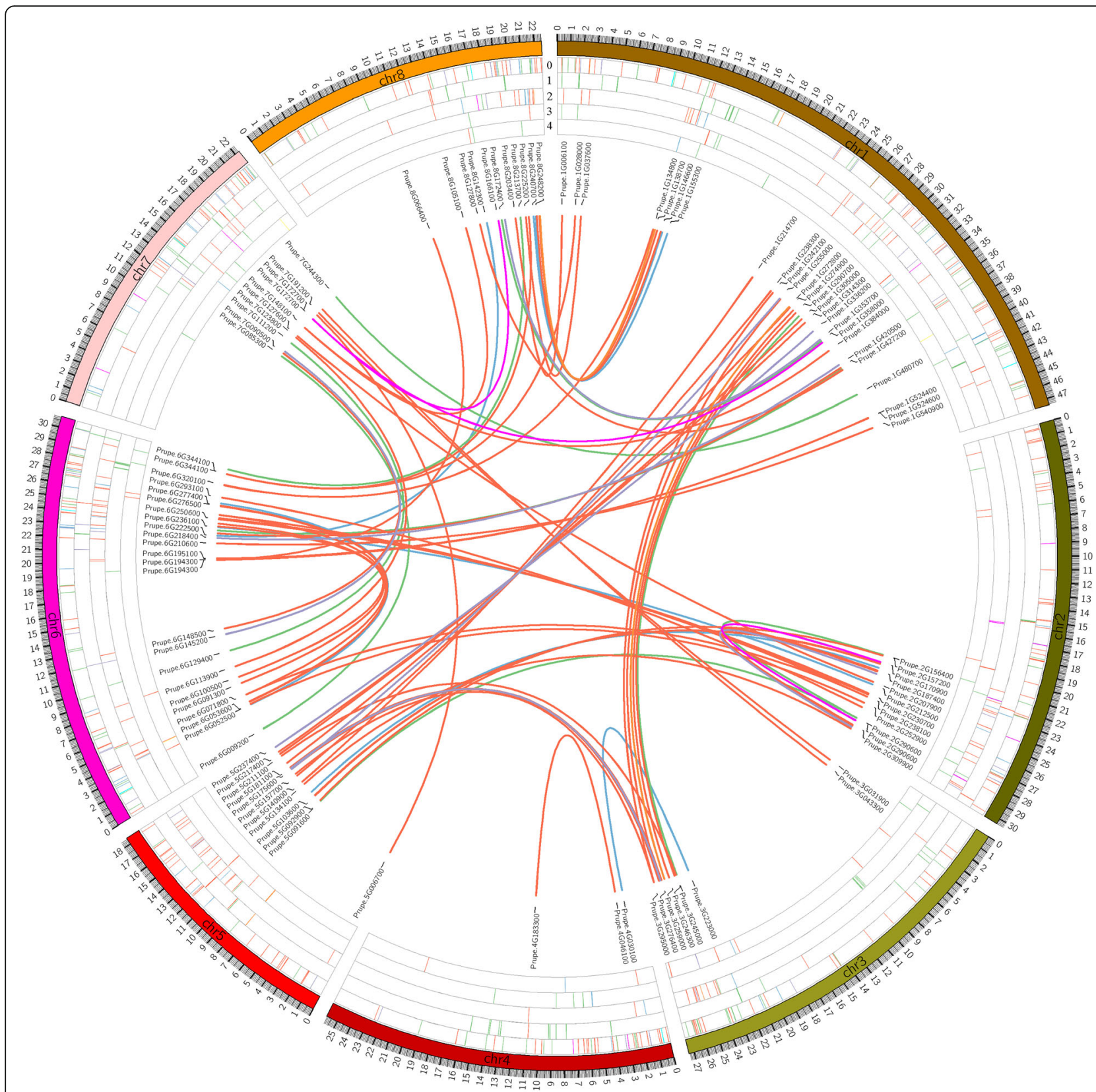

Fig. 1 Genomic distribution and duplication of the PpE3 ligase genes across the 8 chromosomes of peach. The E3 genes are shown with the chromosome on which the occur. Syntenic pairs are linked with lines, with colors representing each subfamily: BTB, blue; Cullin, orange; DDB, yellow; F-box, green; HECT, cyan; RING, red; SKP magenta; and U-box, purple. Each concentric layer represents a type of gene duplication: Layer 0 represents DSD; Layer 1, tandem duplication (TD); Layer 2, whole-genome duplication (WGD); Layer 3, proximal pairs (PD); and Layer 4, singletons

Figure S8; Additional file 1: Table S5). The phylogenetic tree of the HECT subfamily coincided with the classification results using the SMART and Pfam databases (Additional file 2: Figure S9; Additional file 1: Table S5).

\section{Expression of E3 ubiquitin ligase genes during fruit ripening in MF and SH peach}

To reveal the expression patterns of PpE3 ligase genes in peach fruit, the transcriptome of fruit was analyzed during ripening in a MF and a $\mathrm{SH}$ cultivar across four stages of ripening. An average of 37,438,865 paired-end reads were obtained after filtering the reads of low quality and were mapped onto rRNA. About $95.5 \%$ of the high-quality reads were mapped against the peach reference genome (Additional file 1: Table S6).

Among the 515 expressed PpE3 ligase genes, 231 differentially expressed genes (DEGs) were identified at the same-stage between the two peach cultivars (MF vs. $\mathrm{SH}$ ) 


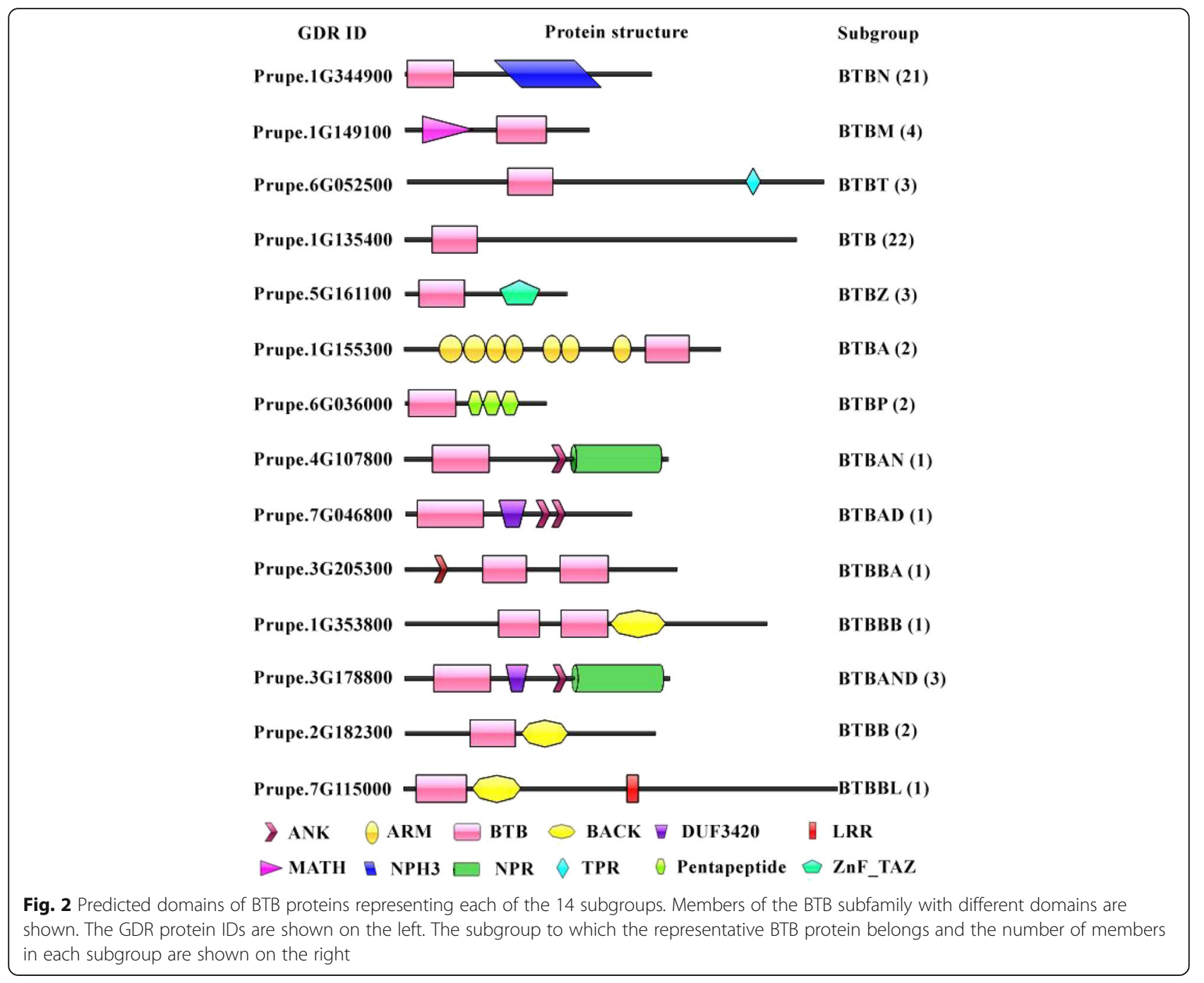

(Table 3; Additional file 1: Table S7). Fifteen randomly selected PpE3 ligase genes and eight genes related to ethylene, auxin and ABA pathway were used to confirm the expression levels by quantitative real-time PCR (qRT-PCR) during fruit ripening in the MF and SH cultivars. The qRT-PCR results were consistent with those of RNA-seq (Fig. 4). The number of DEGs in the different subfamilies was different (Table 3). The number of DEGs was highest for the RING subfamily, while the highest rate of differential expression within a subfamily was for the HECT subfamily.

According to their expression patterns at the same stage of ripening, the 231 DEGs could be classified into eight clusters (Fig. 5). In cluster I, 47 DEGs showed lower expression (1.7- to 42.0-fold) in MF at stage S4III. Twentytwo of the cluster I DEGs belonged to the RING subfamily. Surprisingly, the expression of Prupe.3G223000 was 42.0-fold lower in MF than in SH fruit. Prupe.3G223000 was annotated as a BTB/POZ protein with an NPH3 domain and has high homology with At3G22104 (function unknown). Meanwhile, one F-box gene, Prupe.8G253300 (PpTIR1), which is predicted to function as an auxin receptor TIR1 (Transport inhibitor response1), showed a 3.3-fold lower expression level in MF-S4III than in SHS4III. Prupe.1G097200 (PpXBAT32), in the RING subfamily, has high homolog with AtXBAT32 (At5G57740).

DEGs in cluster II showed higher expression levels in MF peach at almost all analyzed stages of ripening. Twenty genes belonged to this cluster, including two BTB genes, eight F-box genes, nine RING genes and one U-box gene. Prupe.1G020100, a member of the RING subfamily, showed the greatest fold change between the MF and SH peaches (7.2-fold) at stage S4I. Another gene in this cluster, Prupe.8G193500 (PpPUB9), is a U-box gene with a high homology with AtPUB9 (At3G07360).

The transcript abundance of DEGs in cluster III was lower in MF than in $\mathrm{SH}$ at stage S3, but higher in MF at stage S4III. This cluster contained three BTB genes, one F-box gene, eight RING genes and six U-box genes. Prupe.6G222100, a BTB gene, showed the greatest fold 


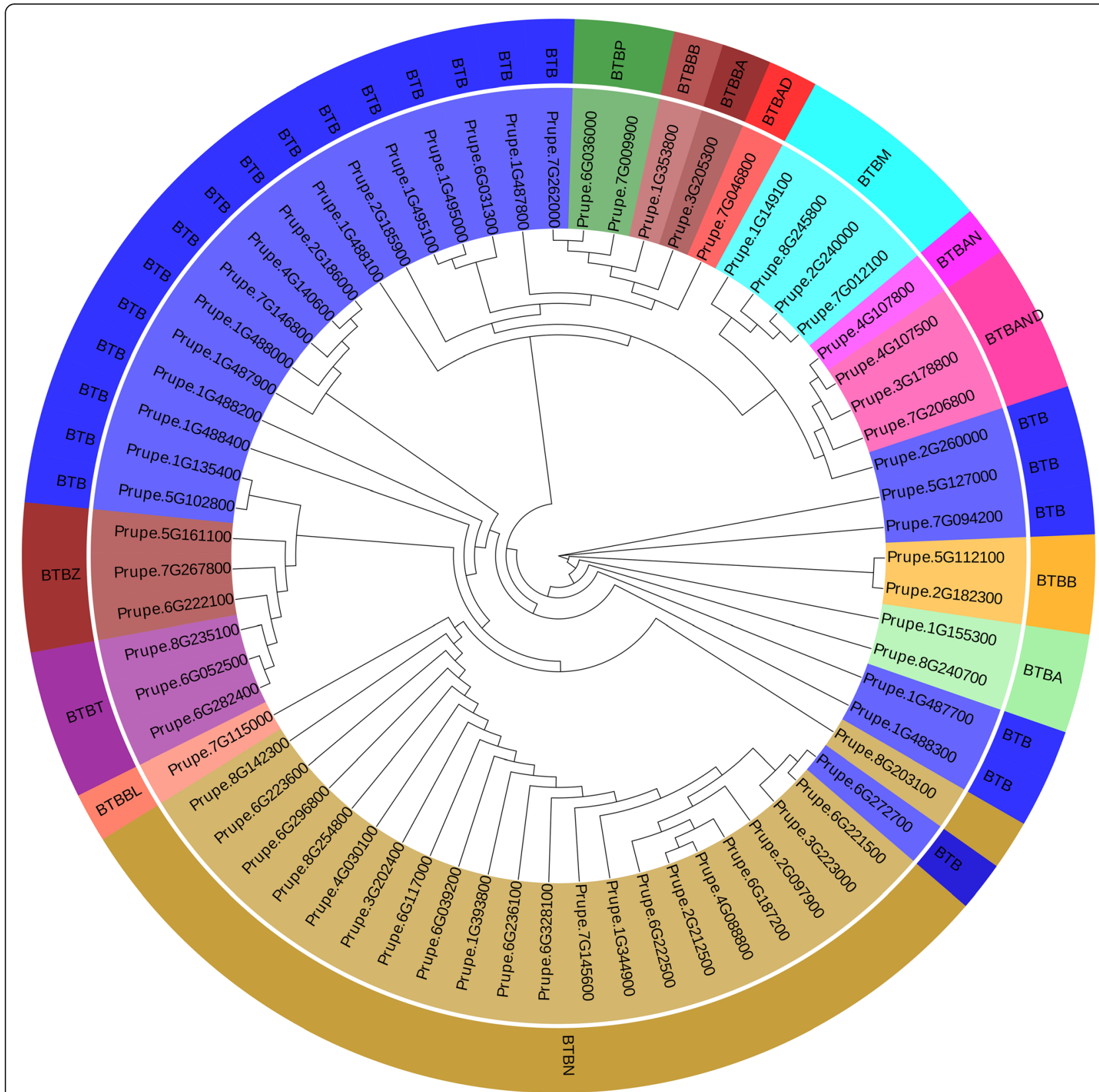

Fig. 3 Phylogenetic analysis of the peach BTB subfamily. The tree was based on the alignment of the full-length BTB protein sequences. The phylogenetic tree was constructed by the MEGA6 program using the neighbor-joining method at 1000 bootstrap replicates

change (-4.6-fold in MF) at stage S3, but a higher expression level in MF at stage S4I. This gene (PpBT4) had high homology with ATBT4 (At5G67480, BTB and TAZ domain protein 4). Prupe.3G125000, a RING gene, showed the greatest fold change (27.0-fold in MF) at stage S4III. Prupe.3G125000 (PpATL43) has high homo$\log$ with ATL43 (At5G05810).

Cluster IV, the second largest group containing 42 genes, showed lower expression in MF at stage S3 but equivalent transcript levels in the other three stages. Their expression at stage 3 was 3.8- to 2.0-fold lower in
MF than in SH. It was notable that four of the five CULLIN DEGs were in this cluster, Prupe.1G138700, Prupe.5G063200, Prupe.5G063700 and Prupe.8G255500. The CULLIN 1 homolog (Prupe.5G063700), which encodes a protein that forms a SCF complex with SKP1 and an F-box protein, showed the maximum fold change (-3.8-fold) in this cluster.

The DEGs in cluster V, the third largest group with 33 genes, showed higher expression levels at stage S4I and lower expression levels at the other stages in the MF fruits. Prupe.5G140900, a RING gene, showed the 
Table 3 Number of expressed genes (EG) and differentially EGs (DEGs) of each subfamily during the peach fruit ripening process

\begin{tabular}{|c|c|c|c|c|c|c|c|c|c|}
\hline \multirow[b]{2}{*}{ Subfamily } & \multicolumn{2}{|l|}{ S3 } & \multicolumn{2}{|l|}{ S4I } & \multicolumn{2}{|l|}{$54 \|$} & \multicolumn{2}{|l|}{ S4III } & \multirow{2}{*}{$\begin{array}{l}\text { DEGs at } \\
\text { least in one } \\
\text { stage }\end{array}$} \\
\hline & $\overline{\mathrm{EG}}$ & DEGs & $\overline{E G}$ & DEGs & $\overline{\mathrm{EG}}$ & DEGs & $\overline{E G}$ & DEGs & \\
\hline RING (338) & $242(71.60 \%)$ & $59(17.46 \%)$ & 244 (72.19\%) & 39 (11.54\%) & $246(72.78 \%)$ & 37 (10.95\%) & 241 (71.30\%) & 69 (20.41\%) & $119(35.21 \%)$ \\
\hline F-box (267) & 122 (45.69\%) & $27(10.11 \%)$ & 127 (47.57\%) & 17 (6.37\%) & $124(46.44 \%)$ & 12 (4.49\%) & 124 (46.44\%) & 34 (12.73\%) & 53 (19.85\%) \\
\hline BTB (67) & $51(76.12 \%)$ & $8(11.94 \%)$ & $50(74.63 \%)$ & 7 (10.45\%) & 54 (80.60\%) & 8 (11.94\%) & 49 (73.13\%) & $13(19.40 \%)$ & 24 (35.82\%) \\
\hline U-box (54) & $37(68.52 \%)$ & $12(22.22 \%)$ & 40 (74.07\%) & $4(7.41 \%)$ & 39 (72.22\%) & $6(11.11 \%)$ & 39 (72.22\%) & 17 (31.48\%) & 23 (42.59\%) \\
\hline SKP (16) & $8(50.00 \%)$ & $1(6.25 \%)$ & 8 (50.00\%) & $0(0.00 \%)$ & 8 (50.00\%) & $1(6.26 \%)$ & 8 (50.00\%) & $1(6.26 \%)$ & $3(18.75 \%)$ \\
\hline Cullin (13) & $9(69.23 \%)$ & $5(38.46 \%)$ & 9 (69.23\%) & $1(7.69 \%)$ & 9 (69.23\%) & $1(7.69 \%)$ & 9 (69.23\%) & $0(0.00 \%)$ & $5(38.46 \%)$ \\
\hline HECT (7) & 7 (100\%) & $1(14.29 \%)$ & 7 (100\%) & $0(0.00 \%)$ & $7(100 \%)$ & $0(0.00 \%)$ & 7 (100\%) & $2(28.57 \%)$ & $3(42.86 \%)$ \\
\hline DDB (3) & $3(100 \%)$ & $0(0.00 \%)$ & $3(100 \%)$ & $0(0.00 \%)$ & $3(100 \%)$ & $0(0.00 \%)$ & $3(100 \%)$ & $1(33.33 \%)$ & 1 (33.33\%) \\
\hline Total (765) & 479 (62.61\%) & 113 (14.77\%) & 488 (63.79\%) & $68(8.89 \%)$ & 490 (64.05\%) & 64 (8.37\%) & 480 (62.75\%) & 137 (17.91\%) & 231 (30.20\%) \\
\hline
\end{tabular}

highest fold change (64.9-fold in MF) at stage S4I but was 1.43-fold lower in MF at both stages S4II and S4III. Prupe.5G140900 had high homology with AtORTH/ VIM (At1G57820, Orthrus/ Variant in methylation). Another RING gene, Prupe.3G242700, showed the greatest reduction in expression $(-8.1$-fold) at stage S4III. The expression of the BTB gene Prupe.8G240700 showed the second lowest fold change (-5.9-fold) in MF compared to $\mathrm{SH}$ at stage S4III.

Genes in cluster VI showed lower expression levels in MF at stages S3 and S4I, but higher expression levels at stages S4II and S4III. Prupe.1G493000 had the greatest swing in expression levels, with the greatest expression reduction (-4.7-fold) at stage S4I and the greatest expression increase (21.1-fold) at stage S4II. Prupe.1G493000 (PpBB) is a RING protein highly homologous to AtBB (At3G63530, AtBIG BROTHER).

Cluster VII included 29 genes, most showing lower expression level in MF peaches at all four stages. Prupe.6G014500 showed the greatest fold change (-26.0fold) at stage S3 and is a U-box gene with high homology to AtPUB11 (At1G23030). However, the function of AtPUB11 is not clear yet.

Cluster VIII contained one BTB, five F-box, nine RING and seven U-box genes that had their highest expression levels in MF at stage S4III. The U-box gene Prupe.8G024500 (PpPUB29, highly homologous to MdPUB29, MDP0000928620) showed the greatest fold change (56-fold) in MF compared to $\mathrm{SH}$ at stage S4III. Among the five F-box genes in this cluster, two genes (Prupe.1G480700 and Prupe.7G244300) were annotated as EBFs (EIN3-binding factor).

\section{Discussion}

The number of E3 families and subfamilies varies greatly between different plant species

E3 ubiquitin ligases are encoded by numerous genes representing different subfamilies in the plant kingdom. The number of putative E3 ligase genes in peach was greater than the number identified in $V$. vinifera (677) but was significantly less than the numbers in six other species (Table 1). PpE3 ligase genes represented almost $3.0 \%$ of the predicted peach genes, higher than in rice, poplar, soybean, Medicago and maize (around 2.2\%), but lower than in Arabidopsis (4.1\%). This indicated that the number of E3 ligase genes in a genome is not associated with the size of the genome.

The number of genes in different subfamilies was also quite different (Table 1). The largest and second largest subfamilies, 338 in the RING and 267 in the F-box subfamilies, accounted for $79 \%$ of the peach E3 ligase genes. The same phenomenon was also seen in grape, maize, polar and soybean (Table 1) [2]. In eight different species, the F-box and RING finger E3 subfamilies are the two most abundant subfamily groups [2]. The number of genes in the DDB and RBX subfamilies was especially small (less than 10) in these eight species. Interestingly, the genes of abundant E3 gene subfamilies were mainly distributed on the longer chromosomes (Chr1 and Chr6), similar to the phenomenon in pear [27]. This indicated that the E3 ligase genes might be non-randomly distributed on the chromosomes of peach.

The exon-intron structure of 354 duplicated F-box genes were analyzed in A. thaliana. There were 137 (38.7\%) F-box genes that carried a different number of exons, while $211(59.6 \%)$ had the same numbers of exons, although the homologous exons were of different length of exons [28]. Duplication of genes allows genetic drift, that includes both alteration of exon-intron structure and the coded amino acids [28]. In this study, the exon-intron number differed in each subfamily. The analysis of exon-intron number might provide more information on the mechanism behind evolution of exonintron structure and distinct biochemical functions.

\section{Different modes of gene duplication occurred within each subfamily}

There are several patterns of gene duplication, with whole-genome duplication (WGD), tandem duplication 

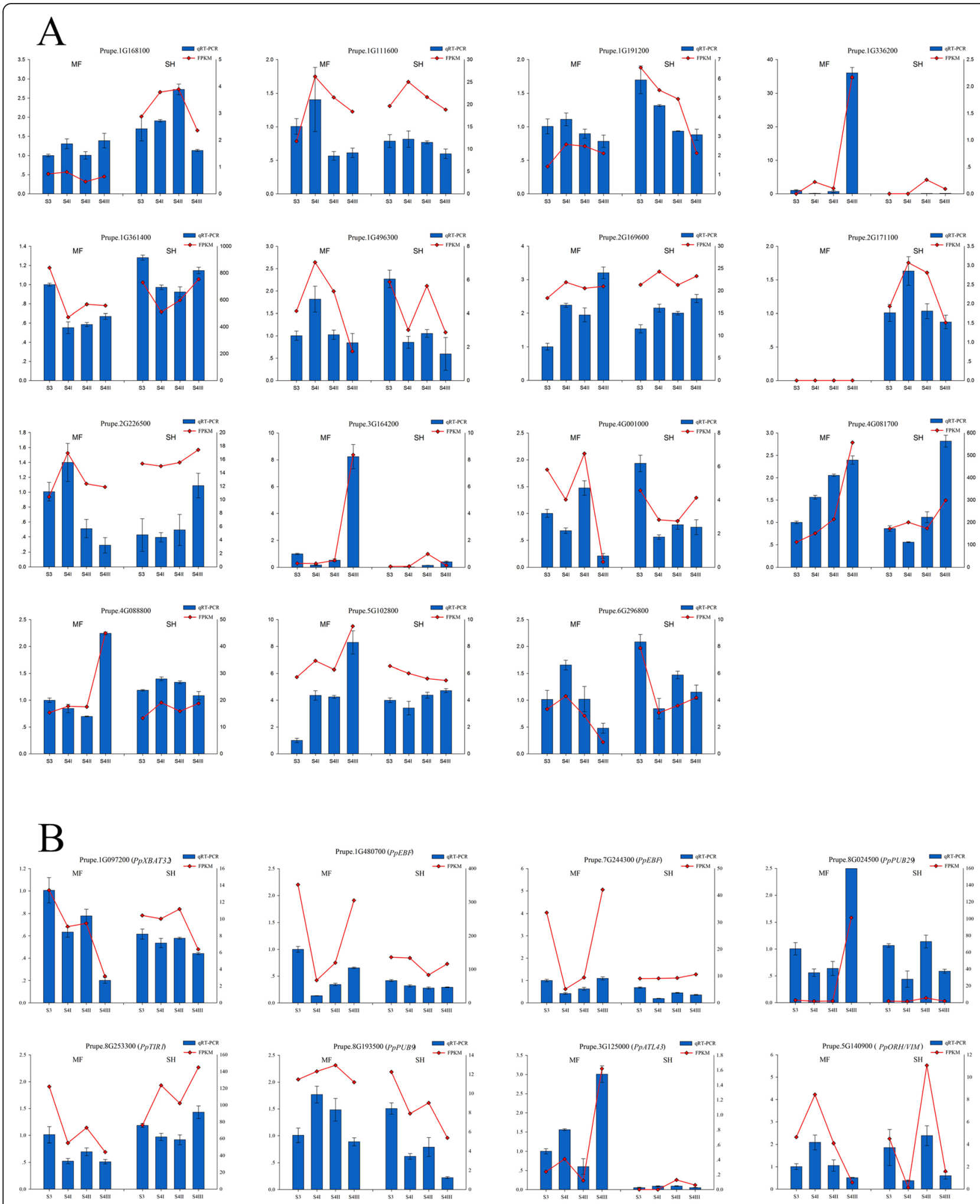

Fig. 4 The relative gene expression of 23 PpE3 ligase genes was analyzed by qRT-PCR in MF and SH at four fruit ripening stages. a The relative gene expression of 15 randomly selected PpE3 genes; $\mathbf{b}$ The relative gene expression of eight genes related to ethylene, auxin and ABA signaling. The left $y$-axis indicates the relative gene expression; the right $y$-axis indicates the FPKM value 


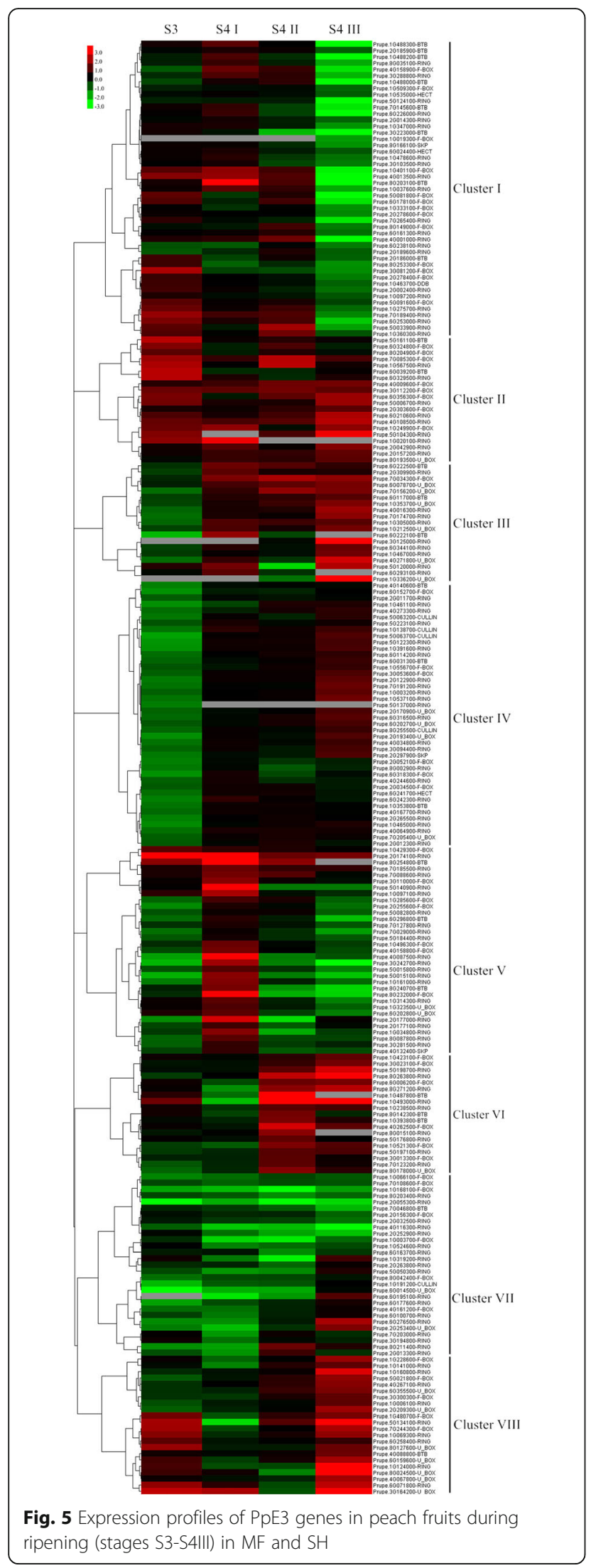

(TD), dispersed gene duplication (DSD) and proximal duplication (PD) being the primary contributors to the expansion of specific gene families in plant genomes [27, 29, 30]. The gene duplication within the different PpE3 subfamilies (Fig. 1, Table 2) showed patterns that were inconsistent with those of other plant species [27, 31-33]. The F-box gene family was expanded in some species through TD and SD [31, 32] and in pear through WGD and DSD [27]. In peach, the data indicated that the F-box family was expanded more through DSD and TD. For the RING gene family in apple, TD and WGD were the major contributors of expansion [33]. In peach, the expansion of the RING subfamily was mainly through DSD and WGD. The same phenomenon was also observed for the BTB and U-Box subfamilies. Based on the above results, DSD is the primary means of gene duplication for the F-box, U-box, RING, BTB and SKP subfamilies in peach. It is most likely that peach has not undergone a recent whole-genome duplication [34].

\section{Classification and domain analysis in F-box, BTB, U-box, RING and HECT subfamilies}

BTB gene family has been discovered in animals and plants $[26,35,36]$. In this study, four new subgroups of BTBs, namely BTBAND, BTBBL, BTBP and BTBAN, were firstly identified in peach. Domains within these new of BTB subgroups were detected in previous studies $[26,35,36]$, but the combinations of the domains are novel. The observation of new subgroups of BTB genes in peach might imply that these genes play new or unknown functions in the growth and development of peach. For instance, the new subgroup BTBAND was a combination of ANK, NPR and DUF domains. It has been documented that ANK (Ankyrin) domains mediate diverse protein-protein interactions [37]. In rice, ANKcontaining proteins participated in various physiological processes, such as responses to NAA (1-naphthylacetic acid) and light treatments [38]. NPR1 (Non-expressor of pathogenesis-related (PR) genes 1) is a master immune regulator [39]. DUF (Domain of unknown function) domains have no characterized function [40]. The new subgroup BTBAND may function by integrating the roles of BTB, ANK, NPR1 and DUF domains. Among the PpE3 ligase genes, the NPH3 (Non-phototropic hypocotyl 3) domain was only observed in the BTB subfamily. During the phototropic response, NPH3 interacts with and mediates the ubiquitination of PHOT1 (Phototropic 1), leading to auxin-regulated differential growth [41]. NPH3 also seems to be specific to higher plants $[42,43]$. We speculated that these BTB genes may play a part in phototropic responses blue light.

The F-box proteins in Arabidopsis, pear and rice have been grouped into 15, 12 and 10 subgroups, respectively $[20,27,29]$. In general, additional domains at the C- 
terminal of an F-box protein recruit specific substrates to the SCF complex and may indicate functional diversification [44]. In peach, 13 F-box proteins were predicted to contain Kelch domains. Genes encoding F-box proteins containing a Kelch domain negatively regulate flavonoid accumulation in rice [45], and in melon [46].

In peach, the U-box proteins were divided into seven subgroups, but the U-box proteins of soybean were grouped into eight subgroups [47]. One PpE3 protein was predicted to contain a TPR domain. The TPR domain interacts with other proteins to form complexes [48]. The TPR1 and TPR2A domains of Hop could specifically recognize the C-terminal heptapeptides of HSP70 and HSP90, respectively [49]. SGT1, which contains a TPR domain and acts as a co-chaperone between HSP90 and SKP1, was proposed to regulate the assembly of SCF complexes [50, 51].

\section{The PpE3 ubiquitin ligase genes exhibit diverse expression patterns during fruit ripening in peach}

The shelf life of peach fruit is greatly affected by the rate of flesh softening, which is a complex process. The molecular and genetic mechanisms regulating flesh softening are unclear. In this study, the transcriptome profiles of the predicted PpE3 ligase genes in MF and $\mathrm{SH}$ peaches were compared during fruit ripening. Of the identified 765 PpE3 ligase genes, 515 (67.32\%) were expressed in fruit flesh, and 231 (30.20\%) were differentially expressed. These DEGs showed different expression levels in the two cultivars when comparing the same stage of ripening, indicating that these E3 ligase genes may be involved in peach fruit softening. Among the eight E3 ligase subfamilies, the RING and F-box subfamilies had greater numbers of DEGs in peach fruit.

There were several DEGs similar to E3 genes known to be involved in ethylene biosynthesis or signal transduction, such as PpXBAT32, PpEBF1s and PpPUB29. In Arabidopsis, the RING-type E3 ligase XBAT32 maintains proper levels of ethylene by targeting ACS4 (1-aminocyclopropane-1-carboxylate synthase 4) and ACS7 for proteasomal degradation [52]. Overexpression of AtEBF1 brings about ethylene insensitivity, which indicates the inverse correlation between EIN3 and the F-box proteins [53]. PpPUB29 is homologous with MdPUB29, which directly ubiquitinates the MdbHLH3 protein, which is related to the regulation of ethylene biosynthesis. MdPUB29 is inhibited by high glucose in apple (Malus domestica) [54]. In this study, the expression level of PpPUB29 was much higher in MF than in $\mathrm{SH}$ at stage S4III. We speculated that PpPUB29 may influence ethylene production in MF.

Auxin regulates many aspects of plant growth and development, such as elongation and fruit development $[12,55]$. Some PpE3s with homology to genes regulated by auxin showed differentially expression in the two types of peach fruit, such as $P p B T 4$ and $P p B B$. In Arabidopsis, the five members of the BTBZ subgroup (AtBT1-AtBT5) showed functional redundancy and transcriptional compensation among the subgroup members and played vital roles in multiple responses and plant development [56-58]. The expression levels of AtBT1, 2, 4, and 5 were enhanced by auxin [56]. The expression of PpBT4 might be regulated by IAA in MF peach. IAA levels increased in MF peach [59], while it did not increase in $\mathrm{SH}$ peach during fruit ripening [12, 60]. In Arabidopsis, AtBB performs a central function in controlling organ size by degrading critical growth stimulators in a dosage-dependent manner [61]. Given the effects of phytohormones on organ growth [62], the higher expression levels of $P p B B$ in MF at stages S4II and S4III might also be due to higher IAA levels in MF. Some components of the ethylene-response machinery are used by auxin signaling to drive ripening $[12,15,60]$. It implied that the functions of AtBB and PpBB might be different in Arabidopsis and peach. We also found that a few DEGs were predicted to function in auxin signal transduction, like TIR1, which is an auxin receptor. In this study, PpTIR1 showed significantly lower expression (3.3-fold) in MF peach compared to in $\mathrm{SH}$ peach at S4III.

Some other DEGs have homologs with known functions that could be related to fruit ripening and softening. A few of E3 ligase genes are homologous to genes involved in ABA signal transduction pathway, such as the RING gene AtATL43, the U-box gene AtPUB9, and the BTB gene AtCRL3, which mediate ubiquitination of ABA receptors [5]. In this study, PpATL43 and PpPUB9 showed higher expression in MF peach at stage S4III. AtATL43 plays a positive role and AtPUB9 a negative role in ABA signaling $[63,64]$. This contrast implies that the signal transduction of ABA might be in a state of dynamic balance. The gene PpORTH/VIM showed much higher expression in MF compared to $\mathrm{SH}$ peach at S4I. The Arabidopsis AtORTH/VIM gene encodes a protein that regulates DNA methylation through it ubiquitin E3 ligase function [65]. These findings were consistent with previous reports that fruit ripening and softening are related to ethylene, IAA, ABA, and DNA methylation $[11,12,19,66]$.

\section{Conclusion}

In this study, 765 putative E3 ubiquitin ligase genes were identified in the peach genome, which could be divided into eight subfamilies according to the characterization of known functional domains. The RBX subfamily was not detected in peach. The primary mode of gene duplication of the PpE3 ligase genes was dispersed gene duplication (DSD). Four new subgroups of the BTB family were first identified in this study. Among the 515 PpE3 
ligase genes expressed during fruit ripening, 231 genes were differentially expressed in $\mathrm{MF}$ and $\mathrm{SH}$ peach varieties at the same stage of fruit ripening. All of the DEGs showed a variety of expression patterns during fruit ripening. Many genes were directly or indirectly related to auxin, ethylene, or ABA biosynthesis or signal transduction and DNA methylation, such as PpXBAT32, PpEBF1s, PpPUB29, PpTIR1, PpPUB9, PpATL43 and $P p O R T H / V I M$. These results inspire us to further explore the roles of these E3 ubiquitin ligase genes in peach fruit ripening and softening to discover the gene regulatory network of this process.

\section{Methods}

\section{Plant materials and RNA-seq}

The two peach (Prunus persica (L.) Batsch) cultivars used in this study have different flesh textures, one a Melting Flesh cultivar named 'Zhongyoutao 13' (MF) and one a Stony Hard cultivar named 'Zhongyoutao 16' $(\mathrm{SH})$. Trees of the two cultivars were planted in an orchard at the Institute of Zhengzhou Fruit Research, Chinese Academy of Agriculture Science (Zhengzhou, China) and were 6-years-old at the time of harvest. Peach fruit were collected at pre-ripening (S3) and ripening (S4 I, S4 II, and S4 III) according to Tonutti et al. [67]. The MF (Zhongyoutao 13) fruits were collected at 87 (S3), 90 (S4 I), 93 (S4 II) and 96 (S4 III) days after full bloom (DAFB). The SH (Zhongyoutao 16) fruits were collected at 79 (S3), 83 (S4 I), 87 (S4 II) and 90 (S4 III) DAFB. Twenty fruits were gathered from five selfpollinated trees at each stage. The fruit mesocarp was separated, immediately frozen in liquid nitrogen, and stored at $-80{ }^{\circ} \mathrm{C}$ for RNA-seq. Eight libraries were generated from the samples of the two peach cultivars at the four stages (S3-S4III) using an Illumina kit and were sequenced by an Illumina $\mathrm{HiSeq}^{\mathrm{TM}} 2500$ sequencer.

\section{Identification of candidate genes and chromosomal distribution analysis}

The deduced protein sequences of the E3 ubiquitin ligase genes from Arabidopsis and grape were downloaded from the NCBI (http://www.ncbi.nlm.nih.gov). The peach genome dataset was downloaded from the Genome Database for Rosaceae (GDR; https://www.rosaceae. org/). Different subfamilies of E3 ubiquitin ligase proteins from Arabidopsis and grape were used as queries to search against the whole peach genome by the BLASTP (http://blast.ncbi.nlm.nih.gov/) program with an E-value $<1 \mathrm{e}-6$. The retrieved sequences were used for domain searches in the Pfam (http://pfam.jouy.inra.fr/) and SMART (http://smart.embl-heidelberg.de/) databases with an E-value cutoff level of 1.0. Candidate genes lacking indispensable E3 domains were removed.
The ubiquitin ligase genes were anchored to peach chromosomes based on the location information extracted from the genome annotation 'Prunus_persica v2.0.a1.gene.gff3' downloaded from the GDR. The physical chromosomal locations and syntenic genes were drafted by Circos software.

The numbers of ubiquitin ligase genes in other plant species were obtained from plantsUPS (a database of the Ubiquitin Proteasome System of plants) [2].

\section{Synteny analysis and dissection of different modes of gene duplication}

The analysis of gene synteny in the peach genome was performed using the Plant Genomic Duplication Database (http:// chibba.agtec.uga.edu/duplication/) [68]. To identify homologous gene pairs, BLASTP was carried out (E-value <1e-5). Gene duplication modes were identified using MCScanX [69].

\section{Classification and phylogenetic analysis}

The potential domains contained in each BTB, F-box, HECT, RING, U-box, Cullin, SKP and DDB sequence were detected by the SMART and Pfam web servers. According to additional domains found in the BTB, F-box and U-box proteins, these genes were grouped into different subgroups. Based on the amino acid residues in conserved positions and the predicted distances between metal ligands, the RING-type for each peach RING domain was determined.

Phylogenetic trees were constructed for the BTB, Fbox, HECT, RING and U-box subfamilies using each full-length protein sequence and the MEGA6 program according to the Neighbor-Joining (NJ) method, with 1000 bootstrap replicates [70].

\section{Expression analysis}

The RNA-seq data of 125-bp paired-ends reads were analyzed to explore the expression patterns of PpE3 ligase genes in peach fruit. The RNA-seq data have been deposited in NCBI under accession number (PRJNA398309). The filtered reads of high quality and no rRNA were mapped to the peach reference genome (https://www. rosaceae.org/species/prunus_persica/genome_v2.0.a1) using TopHat2 [71]. Gene expression levels were measured and expressed in fragments per kilobase of exon per million fragments mapped (FPKM) [72]. Genes with FPKM values $>1$ were defined as showing significant expression. Between $\mathrm{MF}$ and $\mathrm{SH}$ at the same stage, genes were considered to show differential expression at $\log _{2}>2$ and were identified for further analysis. The heatmap of the expression patterns of PpE3 ligase genes was generated by Cluster 3.0 software.

To confirm the differential expression of PpE3 ligase genes identified by RNA-seq, 15 randomly selected PpE3 
ligase genes and eight PpE3 ligase genes with known functions in other plants were analyzed by quantitative real-time PCR (qRT-PCR) at the four stages of ripening in 'Zhongyoutao 13' and 'Zhongyoutao 16'. Total RNA was isolated from peach fruit using the Plant Total RNA Isolation Kit (Sangon, Shanghai, China). The quality and concentration of RNA were detected by $1 \%$ agarose gels and NanoDrop ND-2000 spectrophotometer, respectively. The cDNA was synthesized from RNA with PrimeScript $^{\mathrm{Tu}}$ RT reagent Kit (TaKaRa, Japan), and then diluted for qRT-PCR. qRT-PCR data were normalized using peach RPL13 (Ribosomal Protein L13) gene. The qRT-PCR was performed on a QuantStudio5 system platform (Thremo Lifetech ABI). All primer sequences used in this study are listed in Additional file 1: Table S8. Three biological replicates of each sample were used for qRT-PCR analysis.

\section{Supplementary information}

Supplementary information accompanies this paper at https://doi.org/10. 1186/s12864-019-6258-0.

Additional file 1: Table S1. The characteristics of E3 ligase genes in peach. (XLSX $97 \mathrm{~kb}$ ). Table S2. The distribution of introns among different PpE3s. (XLSX 10 kb). Table S3. Chromosomal distribution of different PpE3 subfamilies in peach. (XLSX $11 \mathrm{~kb}$ ). Table S4. Prevalence of duplication modes in the PpE3 subfamilies. (XLSX 40 kb). Table S5. Domain structure of predicted PpE3 ligase proteins in different subfamilies. (XLSX $108 \mathrm{~kb}$ ). Table S6. The statistical analysis of data by transcriptome sequencing (XLSX $14 \mathrm{~kb}$ ). Table S7. Fold changes in PpE3 ligase gene expression between MF and SH peach fruits at the same stage of ripening. (XLSX 96 kb). Table S8. Primers used in qRT-PCR. (XLSX 14 kb).

Additional file 2: Figure S1. Chromosomal distribution of different PpE3 subfamilies in peach. (JPG $321 \mathrm{~Kb}$ ). Figure S2. Predicted domains of Fbox proteins representing each subgroup. (JPG 1.3 MB). Figure S3. Phylogenetic analysis of the peach F-box subfamily. (JPG 8.1 MB). Figure S4. Predicted domains of U-box proteins representing each subgroup. (JPG 2.0 MB). Figure S5. Phylogenetic analysis of the peach U-box subfamily. (JPG 3.7 MB). Figure S6. Sequence logo of the overrepresented motifs found in the RING-C2, RING-H2, RING-HC, RING-G, RING-V or RING-S/T domains of the RING proteins predicted from the peach genome. (JPG 9.2 MB). Figure S7. Phylogenetic analysis of the peach RING subfamily. (JPG 8.6 MB). Figure S8. Predicted domains of HECT proteins representing each subgroup. (JPG 631.3 kb). Figure S9. Prehylogenetic analysis of the peach HECT subfamily. (JPG $920.5 \mathrm{~kb}$ ).

\section{Abbreviations}

ABA: Abscisic Acid; ACS: 1-aminocyclopropane-1- carboxylate synthases; ANK: Ankyrin; ARM: Armadillo; BTB: Broad-complex, tramtrack and bric-a-brac; DAFB: Days after full bloom; DDB: Damaged DNA binding protein; DEG: Differentially expressed gene; DSD: Dispersed gene duplication; DUF: Domain of unknown function; EBF: EIN3-binding factor; FPKM: Fragments per kilobase of exon per million fragments mapped; GDR: Genome database for Rosaceae; HECT: Homologous to the E6associated protein carboxyl terminus; KAP: Kinesin-associated protein; MF: Melting flesh; NAA: 1-naphthylacetic acid; NCBI: National Center for Biotechnology Information; NJ: Neighbor-Joining; NMF: Non-melting flesh; NPH3: Non-phototropic hypocotyl 3; NPR1: Non-expressor of pathogenesisrelated (PR) genes 1; ORTHNIM: Orthrus/Nariant in methylation; PD: Proximal pairs; PHOT1: Phototropic 1; PUB: Plant U-box; qRT-PCR: Quantitative RealTime PCR; RING: Really interesting new gene; SCF: SKP-Cullin-F-box; SH: Stony hard; TD: Tandem duplication; TIR1: Transport inhibitor response 1; TPR: Tetratrico peptide repeat; UBA: Ub-associated; UFD2: Ubiquitin fusion degradation 2; UGT: UDP-glycosyltransferases; UIM: Ub-interacting motif;
WGD: Whole-genome duplication; XBAT32: XB3 ortholog 2 in Arabidopsis thaliana

\section{Acknowledgements}

We thank Anita K. Snyder for critical reading and language editing of the manuscript.

\section{Authors' contributions}

$J F, B T$ and $X L$ conceived and designed the experiments. WZ provided plant material. BT, $W Z$ and $L Z$ performed the experiments. $X L, J C$ and $W W$ performed the bioinformatics analyses. $X Z$ and $X Y$ analyzed the data. $J L$ and $\mathrm{ZL}$ contributed analytical tools. BT and XL wrote the paper. All authors contributed, read and approved the final manuscript.

\section{Funding}

This project was completed at the Henan Provincial Key Laboratory of Fruit and Cucurbit Biology and was supported by Major Science and Technology Project in Henan Province (151100110900), the Special Fund for Henan Agriculture Research System (S2014-11-G02), Henan Scientific and Technological Research Project (172102410049) and Innovation Team Project of Henan University (19IRTSTHN009). The funding bodies had no role in the design of the study, collection, analysis, or interpretation of data or in the writing of the manuscript.

\section{Availability of data and materials}

All data presented in this study are provided either in the manuscript or additional files. Raw reads data has been deposited in NCBI under accession number PRJNA398309.

\section{Ethics approval and consent to participate}

The peach cultivars 'Zhongyoutao 13' and 'Zhongyoutao 16' used in this study were planted and grown in Zhengzhou, Henan Province, China. Those two cultivars were widely planted in Henan Province. This study did not require ethics approval and consent as wild, no endangered or protected plant species were involved.

\section{Consent for publication}

Not applicable.

\section{Competing interests}

The authors declare that they have no competing interests.

\section{Author details}

${ }^{1}$ College of Horticulture, Henan Agricultural University, Zhengzhou 450002, China. ${ }^{2}$ Henan Key Laboratory of Fruit and Cucurbit Biology, Zhengzhou 450002, China. ${ }^{3}$ Zhengzhou Fruit Research Institute, Chinese Academy of Agricultural Sciences, Zhengzhou 450009, China.

Received: 1 May 2019 Accepted: 31 October 2019

Published online: 21 November 2019

\section{References}

1. Wang F, Deng XW. Plant ubiquitin-proteasome pathway and its role in gibberellin signaling. Cell Res. 2011;21(9):1286-94.

2. Du Z, Zhou X, Li L, Su Z. PlantsUPS: a database of plants' ubiquitin proteasome system. BMC Genomics. 2009;10(1):227.

3. Moon J, Parry G, Estelle M. The ubiquitin-proteasome pathway and plant development. Plant Cell. 2004;16(12):3181-95.

4. Santner A, Estelle M. The ubiquitin-proteasome system regulates plant hormone signaling. Plant J. 2010;61(6):1029-40.

5. Yu F, Wu Y, Xie Q. Ubiquitin-proteasome system in ABA signaling: from perception to action. Mol Plant. 2016;9(1):21-33.

6. Ali M, Uemura T, Ramadan A, Adachi K, Nemoto K, Nozawa A, et al. The Ring-type E3 ubiquitin ligase JUL1 targets the VQ-motif protein JAV1 to coordinate jasmonate signaling. Plant Physiol. 2019;179(4):1273-84.

7. Lim CW, Baek W, Lee SC. The pepper Ring-type E3 ligase CaAIRF1 regulates $A B A$ and drought signaling via CaADIP1 protein phosphatase degradation. Plant Physiol. 2017;173(4):2323-39.

8. Haji T, Yaegaki H, Yamaguchi M. Changes in Ethylene production and flesh firmness of melting, nonmelting and stony hard peaches after harvest. J Japan Soc Hort Sci. 2001;70(4):458-9. 
9. Peace $\mathrm{CP}$, Crisosto $\mathrm{CH}$, Gradziel TM. Endopolygalacturonase: a candidate gene for freestone and melting fleshin peach. Mol Breed. 2005;16(1):21-31

10. Ghiani A, Onelli E, Aina R, Cocucci M, Citterio S. A comparative study of melting and non-melting flesh peach cultivars reveals that during fruit ripening endo-polygalacturonase (endo-PG) is mainly involved in pericarp textural changes, not in firmness reduction. J Exp Bot. 2011;62(11):4043-54.

11. Gu C, Wang L, Wang W, Zhou H, Ma B, Zheng H, et al. Copy number variation of a gene cluster encoding endopolygalacturonase mediates flesh texture and stone adhesion in peach. J Exp Bot. 2016;67(6):1993-2005.

12. Pan L, Zeng W, Niu L, Lu Z, Liu H, Cui G, et al. PpYUC11, a strong candidate gene for the stony hard phenotype in peach (prunus persica L. Batsch), participates in IAA biosynthesis during fruit ripening. J Exp Bot. 2015;66(22): 7031-44.

13. Zeng W, Pan L, Liu H, Niu L, Lu Z, Cui G, et al. Characterization of 1aminocyclopropane-1-carboxylic acid synthase (ACS) genes during nectarine fruit development and ripening. Tree Genet Genom. 2015;11(2):18.

14. Hayama $\mathrm{H}$, Tatsuki M, Ito A, Kashimura $Y$. Ethylene and fruit softening in the stony hard mutation in peach. Postharvest Biol Technol. 2006;41(1):16-21.

15. Tatsuki M, Soeno K, Shimada Y, Sawamura Y, Suesada Y, Yaegaki H, et al. Insertion of a transposon-like sequence in the $5^{\prime}$-flanking region of the YUCCA gene causes the stony hard phenotype. Plant J. 2018;96(4):815-27.

16. Soto A, Ruiz KB, Ravaglia D, Costa G, Torrigiani P. ABA may promote or delay peach fruit ripening through modulation of ripening- and hormonerelated gene expression depending on the developmental stage. Plant Physiol Biochem. 2013;64:11-24.

17. Sun $L$, Sun $Y$, Zhang M, Wang L, Ren J, Cui M, et al. Suppression of 9-cisepoxycarotenoid dioxygenase, which encodes a key enzyme in abscisic acid biosynthesis, alters fruit texture in transgenic tomato. Plant Physiol. 2012; 158(1):283-98

18. Giribaldi M, Geny L, Delrot S, Schubert A. Proteomic analysis of the effects of $A B A$ treatments on ripening vitis vinifera berries. J Exp Bot. 2010;61(9): 2447-58.

19. Wang $X$, Zeng W, Ding $Y$, Wang $Y$, Niu L, Yao J, et al. PpERF3 positively regulates $A B A$ biosynthesis by activating $P p N C E D 2 / 3$ transcription during fruit ripening in peach. Horticulture Research. 2019;6(1):19.

20. Jain M, Nijhawan A, Arora R, Agarwal P, Ray S, Sharma P, et al. F-box proteins in rice. Genome-wide analysis, classification, temporal and spatial gene expression during panicle and seed development, and regulation by light and abiotic stress. Plant Physiol. 2007;143(4):1467-83.

21. Xu J, Xing S, Cui H, Chen X, Wang X. Genome-wide identification and characterization of the apple (Malus domestica) HECT ubiquitin-protein ligase family and expression analysis of their responsiveness to abiotic stresses. Mol Gen Genomics. 2016;291(2):635-46.

22. Jia Q, Xiao ZX, Wong FL, Sun S, Liang KJ, Lam HM. Genome-wide analyses of the soybean F-box gene family in response to salt stress. Int J Mol Sci. 2017;18(4):818

23. Samach A, Klenz JE, Kohalmi SE, Risseeuw E, Haughn GW, Crosby WL. The UNUSUAL FLORAL ORGANS gene of Arabidopsis thaliana is an F-box protein required for normal patterning and growth in the floral meristem. Plant J. 1999;20(4):433-45.

24. Wang H, Pan J, Li Y, Lou D, Hu Y, Yu D. The DELLA-CONSTANS transcription factor cascade integrates gibberellic acid and photoperiod signaling to regulate flowering. Plant Physiol. 2016;172(1):479-88.

25. Seo Kl, Lee JH, Nezames CD, Zhong S, Song E, Byun MO, et al. ABD1 is an Arabidopsis DCAF substrate receptor for CUL4-DDB1-based E3 ligases that acts as a negative regulator of abscisic acid signaling. Plant Cell. 2014;26(2):695-711.

26. Li J, Su X, Wang Y, Yang W, Pan Y, Su C, et al. Genome-wide identification and expression analysis of the BTB domain-containing protein gene family in tomato. Genes Genomics. 2017:40(1):1-15.

27. Wang GM, Yin H, Qiao X, Tan X, Gu C, Wang BH, et al. F-box genes: genome-wide expansion, evolution and their contribution to pollen growth in pear (Pyrus bretschneideri). Plant Sci. 2016;253:164-75.

28. Xu G, Guo C, Shan H, Kong H. Divergence of duplicate genes in exon-intron structure. Proc Natl Acad Sci U S A. 2012;109(4):1187-92.

29. Kuroda H, Takahashi N, Shimada H, Seki M, Shinozaki K, Matsui M. Classification and expression analysis of Arabidopsis F-box-containing protein genes. Plant Cell Physiol. 2002;43(10):1073-85.

30. Liu Q, Yang J, Wang Z, Xu X, Mao X, Li D, et al. Genome-wide classification, identification and expression profile of the C3HC4-type Ring finger gene family in poplar (Populus trichocarpa). Plant Mol Biol Report. 2015;33(6): $1740-54$.
31. Yang X, Kalluri UC, Jawdy S, Gunter LE, Yin T, Tschaplinski TJ, et al. The Fbox gene family is expanded in herbaceous annual plants relative to woody perennial plants. Plant Physiol. 2008;148(3):1189-200.

32. Xu G, Ma H, Nei M, Kong H. Evolution of F-box genes in plants: different modes of sequence divergence and their relationships with functional diversification. Proc Natl Acad Sci U S A. 2009;106(3):835-40.

33. Li Y, Wu B, Yu Y, Yang G, Wu C, Zheng C. Genome-wide analysis of the Ring finger gene family in apple. Mol Gen Genomics. 2011;286(1):81-94.

34. International Peach Genome I, Verde I, Abbott AG, Scalabrin S, Jung S, Shu $S$, et al. The high-quality draft genome of peach (Prunus persica) identifies unique patterns of genetic diversity, domestication and genome evolution. Nat Genet. 2013;45(5):487-94.

35. Cheng D, Qian W, Meng M, Wang Y, Peng J, Xia Q. Identification and expression profiling of the BTB domain-containing protein gene family in the silkworm, bombyx mori. Int J Genomics. 2014;2014:865065.

36. Stogios PJ, Downs GS, Jauhal JJ, Nandra SK, Prive GG. Sequence and structural analysis of BTB domain proteins. Genome Biol. 2005;6(10):R82.

37. Sedgwick SG, Smerdon SJ. The ankyrin repeat: a diversity of interactions on a common structural framework. Trends Biochem Sci. 1999;24(8):311-6.

38. Huang J, Zhao X, Yu H, Ouyang Y, Wang L, Zhang Q. The ankyrin repeat gene family in rice: genome-wide identification, classification and expression profiling. Plant Mol Biol. 2009;71(3):207-26.

39. Withers J, Dong X. Posttranslational modifications of NPR1: a single protein playing multiple roles in plant immunity and physiology. PLoS Pathog. 2016;12(8):e1005707.

40. Bateman A, Coggill P, Finn RD. DUFs: families in search of function. Acta Crystallographica Section F Structural Biology and Crystallization Communications. 2010;66(10):1148-52.

41. Holland JJ, Roberts D, Liscum E. Understanding phototropism: from Darwin to today. J Exp Bot. 2009;60(7):1969-78.

42. Motchoulski A. Arabidopsis NPH3: a NPH1 photoreceptor-interacting protein essential for phototropism. Science. 1999:286(5441):961-4.

43. Roberts D, Pedmale UV, Morrow J, Sachdev S, Lechner E, Tang X, et al. Modulation of phototropic responsiveness in Arabidopsis through ubiquitination of phototropin 1 by the CUL3-Ring E3 ubiquitin ligase CRL3(NPH3). Plant Cell. 2011;23(10):3627-40.

44. Gagne JM, Downes BP, Shiu SH, Durski AM, Vierstra RD. The F-box subunit of the SCF E3 complex is encoded by a diverse superfamily of genes in Arabidopsis. Proc Natl Acad Sci U S A. 2002;99(17):11519-24.

45. Shao T, Qian Q, Tang D, Chen J, Li M, Cheng Z, et al. A novel gene IBF1 is required for the inhibition of brown pigment deposition in rice hull furrows. Theor Appl Genet. 2012;125(2):381-90.

46. Feder A, Burger J, Gao S, Lewinsohn E, Katzir N, Schaffer AA, et al. A kelch domain-containing F-box coding gene negatively regulates flavonoid accumulation in muskmelon. Plant Physiol. 2015;169(3):1714-26.

47. Wang N, Liu Y, Cong Y, Wang T, Zhong X, Yang S, et al. Genome-wide identification of soybean U-box E3 ubiquitin ligases and roles of GmPUB8 in negative regulation of drought stress response in Arabidopsis. Plant Cell Physiol. 2016;57(6):1189-209.

48. Lamb JR. Tetratrico peptide repeat interactions: to TPR or not to TPR? Trends Biochem Sci. 1995;20(7):257-9.

49. Scheufler C, Brinker A, Bourenkov G, Pegoraro S, Moroder L, Bartunik H, et al Structure of TPR domain-peptide complexes. Cell. 2000;101(2):199-210.

50. Catlett MG, Kaplan KB. Sgt1p is a unique co-chaperone that acts as a client adaptor to link HSP90 to SKP1p. J Biol Chem. 2006;281(44):33739-48.

51. Boter M, Amigues B, Peart J, Breuer C, Kadota Y, Casais C, et al. Structural and functional analysis of SGT1 reveals that its interaction with HSP9O is required for the accumulation of $\mathrm{Rx}$, an $\mathrm{R}$ protein involved in plant immunity. Plant Cell. 2007:19(11):3791-804.

52. Lyzenga WJ, Booth JK, Stone SL. The Arabidopsis Ring-type E3 ligase XBAT32 mediates the proteasomal degradation of the ethylene biosynthetic enzyme, 1-aminocyclopropane-1-carboxylate synthase 7. Plant J. 2012;71(1): 23-34.

53. Potuschak T, Lechner E, Parmentier Y, Yanagisawa S, Grava S, Koncz C, et al. EIN3-dependent regulation of plant ethylene hormone signaling by two Arabidopsis F box proteins : EBF1 and EBF2. Cell. 2003;115(6):679-89.

54. Hu DG, Yu JQ, Han PL, Xie XB, Sun CH, Zhang QY, et al. The regulatory module MdPUB29-MdbHLH3 connects ethylene biosynthesis with fruit quality in apple. New Phytol. 2019;221(4):1966-82.

55. Depuydt $\mathrm{S}$, Hardtke CS. Hormone signalling crosstalk in plant growth regulation. Curr Biol. 2011;21(9):R365-73. 
56. Robert HS, Quint A, Brand D, Vivian-Smith A, Offringa R. BTB and TAZ domain scaffold proteins perform a crucial function in Arabidopsis development. Plant J. 2009;58(1):109-21.

57. Du LQ, Poovaiah BW. A novel family of $\mathrm{Ca}^{2+} /$ calmodulin-binding proteins involved in transcriptional regulation: interaction with fsh/Ring3 class transcription activators. Plant Mol Biol. 2004;54(4):549-69.

58. Mandadi KK, Misra A, Ren S, McKnight TD. BT2, a BTB protein, mediates multiple responses to nutrients, stresses, and hormones in Arabidopsis. Plant Physiol. 2009;150(4):1930-9

59. Miller AN, Walsh CS, Cohen JD. Measurement of indole-3-acetic acid in peach fruits (Prunus persica L. Batsch cv Redhaven) during development. Plant Physiol. 1987;84:491-4.

60. Tatsuki M, Nakajima N, Fujii H, Shimada T, Nakano M, Hayashi K, et al. Increased levels of IAA are required for system 2 ethylene synthesis causing fruit softening in peach (Prunus persica L. Batsch). J Exp Bot. 2013;64(4): 1049-59.

61. Disch S, Anastasiou E, Sharma VK, Laux T, Fletcher JC, Lenhard M. The E3 ubiquitin ligase BIG BROTHER controls Arabidopsis organ size in a dosagedependent manner. Curr Biol. 2006;16(3):272-9.

62. Mizukami Y. A matter of size: developmental control of organ size in plants. Curr Opin Plant Biol. 2001;4(6):533-9.

63. Serrano M, Parra S, Alcaraz LD, Guzmán P. The ATL gene family from Arabidopsis thaliana and Oryza sativa comprises a large number of putative ubiquitin ligases of the Ring-H2 type. J Mol Evol. 2006;62(4):434-45.

64. Samuel MA, Mudgil Y, Salt JN, Delmas F, Ramachandran S, Chilelli A, et al. Interactions between the S-domain receptor kinases and AtPUB-ARM E3 ubiquitin ligases suggest a conserved signaling pathway in Arabidopsis. Plant Physiol. 2008;147(4):2084-95.

65. Kraft E, Bostick M, Jacobsen SE, Callis J. ORTH/VIM proteins that regulate DNA methylation are functional ubiquitin E3 ligases. Plant J. 2008;56(5):70415

66. Gallusci P, Hodgman C, Teyssier E, Seymour GB. DNA methylation and chromatin regulation during fleshy fruit development and ripening. Front Plant Sci. 2016;7:807.

67. Tonutti P, Bonghi C, Ruperti B, Tornielli GB, Ramina A. Ethylene evolution and 1-aminocyclopropane-1-carboxylate oxidase gene expression during early development and ripening of peach fruit. Am Soc Horticult Sci. 1997; 122(5):642-7.

68. Lee T, Tang H, Wang X, Paterson AH. PGDD: a database of gene and genome duplication in plants. Nucleic Acids Res. 2012;41(D1):D1152-8,

69. Wang Y, Tang H, Debarry JD, Tan X, Li J, Wang X et al: MCScanX: a toolkit for detection and evolutionary analysis of gene synteny and collinearity. Nucleic Acids Res 2012; 40(7):e49-e49.

70. Tamura K, Stecher G, Peterson D, Filipski A, Kumar S. MEGA6: molecular evolutionary genetics analysis version 6.0. Mol Biol Evol. 2013;30(12):2725-9.

71. Kim D, Pertea G, Trapnell C, Pimentel H, Kelley R, Salzberg SL. TopHat2: accurate alignment of transcriptomes in the presence of insertions, deletions and gene fusions. Genome Biol. 2013;14(4):R36.

72. Trapnell C, Williams BA, Pertea G, Mortazavi A, Kwan G, van Baren MJ, et al. Transcript assembly and quantification by RNA-seq reveals unannotated transcripts and isoform switching during cell differentiation. Nat Biotechnol. 2010;28(5):511-5.

\section{Publisher's Note}

Springer Nature remains neutral with regard to jurisdictional claims in published maps and institutional affiliations.

Ready to submit your research? Choose BMC and benefit from:

- fast, convenient online submission

- thorough peer review by experienced researchers in your field

- rapid publication on acceptance

- support for research data, including large and complex data types

- gold Open Access which fosters wider collaboration and increased citations

- maximum visibility for your research: over $100 \mathrm{M}$ website views per year

At BMC, research is always in progress.

Learn more biomedcentral.com/submissions 